\title{
Assessment of inter- and intra-operator cephalometric tracings on cone beam CT radiographs: comparison of the precision of the cone beam CT versus the latero-lateral radiograph tracing
}

\author{
Giampietro Farronato*, Sara Salvadori, Francesca Nolet, Alessandro Zoia and Davide Farronato
}

\begin{abstract}
Background: In this study we aimed at quantifying the possible errors which may occur when assessing specific reference planes and linear derivants on cephalometric radiographs traced manually and digitally. Furthermore, we have compared the precision of the tracings according to both the two- and three-dimensional ( $2 \mathrm{D}$ and 3D respectively) techniques and between clinicians.

Findings: We have obtained via cone beam computed tomography (CBCT) archive of the orthodontics department of the University of Milan 20 cone beam $C T$ radiographs from which we have obtained 20 latero-lateral radiographs. Five independent clinicians referred to as A, B, C, D, E have been randomly selected to trace both radiographs maintaining the same working and lighting conditions to minimize the possibility of operator- and environment-dependent errors from occurring. The results have been statistically assessed by Student's $t$ test. The comparison of the data gathered from the tracings in 2D and 3D shows that certain measurements have statistically significant differences. Particularly, the difference in the measurements of the sagittal dimension of the mandible and the anterior and posterior nasal spines has resulted to be statistically significant. The results of the intra-operator comparison proved that the 3D technique is extremely precise.

Conclusion: Our study determines that the 3D technique allows to obtain more precise results and with several advantages when compared to the conventional technique such as a true representation of the anatomical structures, less risk of errors occurring due to clinician skills and absence of overlapping anatomical structures.
\end{abstract}

Keywords: Cephalometric assessment; Cone beam computer tomography; Latero-lateral teleradiography

\section{Findings}

\section{Introduction}

Conventional cephalometry has been used since the early 1930s as the standard procedure to assess discrepancies in the dento-alveolar and skeletal relationships. It is widely used to assess the changes that occur post-treatment and to evaluate growth [1-5]. The three X-ray projections onto which the traditional cephalometric analysis is based are the postero-anterior teleradiography, the axial projection and the latero-lateral teleradiography. However, the conventional cephalometric approach encounters several limitations such as it is a two-dimensional (2D) representation of the three-dimensional (3D) structures [6-9]. Therefore, the reliability of the cephalometric analyses depends on the correct projection and identification of errors [6-9].

To avoid such problems, cone beam computed tomography $(\mathrm{CBCT})$ has been successfully introduced and used in dentistry as it represents the true 3D morphology of the skeletal structures of the cranium [10-16]. In addition, $\mathrm{CBCT}$ has a reduced radiation exposure when compared

* Correspondence: giampietro.farronato@unimi.it

Orthodontics Department, University of Milan, Milan, Italy 
to multislice CT and it can also be used to assess orthodontic patients $[17,18]$.

In this study we assessed the number of possible errors that may occur when tracing cephalometric radiographs using the three-dimensional technique of the University of Milan compared with the precision of the radiographic position of linear measurements obtained via the tracings of the two-dimensional radiographs. We also assessed the reliability of the clinicians as we compared their own tracings after 2 months.

To carry out such assessment, we compared the precision in which different clinicians and the same clinician, but at different times, obtained specific cephalometric measurements according to the method used (either the two-dimensional or three-dimensional technique).

\section{Materials and methods}

Twenty cone beam CT radiographs have been selected and from these, 20 corresponding latero-lateral teleradiographs have been obtained (two-dimensional technique) with the use of the software Mimics ${ }^{\bullet}$ Materialise (Materialise HQ, Leuven, Belgium). The cases have been randomly selected from the archives of the orthodontics department of the University of Milan. The patients were 12 females and eight males and their ages ranged from 8 to 16 years (mean $12.9 \pm 1.7$ years). The patients have been informed of the research and authorization has been obtained from both patients and parents.

To decrease the risk of possible clinician-dependent errors occurring during the tracing of the cephalometric radiographs, we have maintained the same specific working conditions for each clinician. For instance, the clinicians had the same training and were all graduates from the University of Milan and hence had similar levels of understanding of the principles of cephalometric analyses.

All patients were undergoing treatment at the orthodontic department of the Dentistry University of Milan and all had had a cone beam CT radiograph using the I-Cat Classic ${ }^{\bullet}$ system (Imaging Science International, Hatfield, PA, USA).

The manual tracing of the traditional latero-lateral teleradiographs was performed on fine grain $0.003 \mathrm{in}$. transparent acetate papers (orthotrace; Rocky Mountains Orthodontics, Denver, CO, USA) using a $0.3-\mathrm{mm}$ lead pencil. The Unitek cephalometric protractor (Unitek, Allerum, Sweden) has been used that has a standard resolution of $1 \mathrm{~mm}$ and $1^{\circ}$.

The tracing process was conducted in a darkened room using a screen viewing box.

The CBCT radiographs of the selected patients have been assessed according to the three-dimensional cephalometric analyses of the University of Milan using the software Mimics ${ }^{\oplus}$ Materialise.
Six specific reference planes have been selected and assessed which were the following:

- S-N: orientation of the anterior cranial base

- SNP-A: sagittal dimension of the upper maxillary process

- GO-ME: sagittal dimension of the mandible

- N-SNA: anterior superior vertical dimension

- SNA-ME: anterior inferior vertical dimension

- N-ME: total anterior vertical dimension

The study was carried out in July to August 2013 at (time $1=\mathrm{T} 1$ ) by five selected clinicians which we will refer to as A, B, C, D and E. The clinicians were randomly selected and were all orthodontists working in the orthodontics department of the University of Milan who had graduated from the University of Milan. Each has manually traced 20 cephalometric radiographs and measured six reference planes according to the twodimensional technique. The same clinicians then traced, according to the three-dimensional cephalometric technique, the corresponding 20 cone beam CT radiographs.

After 2 months (time $2=\mathrm{T} 2$ ), the five clinicians (A, B, $C, D, E)$ have repeated the same tracings using both the two-dimensional and three-dimensional techniques and they re-recorded, for each radiograph, the same six reference planes.

The statistical analyses of the data have been measured using the Student's $t$ test and statistical significance was set at $p<0.05$. Confidence intervals were determined at $95 \%$.

The data has been inserted into the statistical programme SPSS $^{\circ}$ statistical package 17.00 for Windows (IBM Corporation, Sommers, NY, USA).

\section{Results}

Below is the comparison of the measurements obtained by the different clinicians at T1 and T2 according to both 2D and 3D techniques (Table 1) and the comparison of the tracings according to the $3 \mathrm{D}$ and $2 \mathrm{D}$ techniques by the same clinician at different times. The reference planes, which appeared to vary more frequently, were the anterior and posterior nasal spines and the GO-ME plane (Figure 1).

\section{Patient GA}

The 3D versus 2D comparison assessed by Student's $t$ statistical test where $p<0.05$ proves to be significant for the reference planes GO-ME, SNA-ME and N-ME. The $3 \mathrm{D}$ versus 3D comparison assessed by Student's $t$ test where $p<0.05$ was not proved to be statistically significant. The $2 \mathrm{D}$ versus $2 \mathrm{D}$ comparison was not proved to be statistically significant. 
Table 1 Summary of the results of the comparison of 3D with 2D techniques

\begin{tabular}{|c|c|c|c|c|c|c|}
\hline \multirow[t]{2}{*}{ Patient } & \multicolumn{6}{|c|}{ Reference planes } \\
\hline & $\overline{S-N}$ & SNP-A & GO-ME & N-SNA & SNA-ME & $\overline{\mathrm{N}-\mathrm{ME}}$ \\
\hline$\overline{\mathrm{GA}}$ & & & $x$ & & $x$ & $x$ \\
\hline $\mathrm{BM}$ & & $x$ & $x$ & $x$ & & \\
\hline CF & & $x$ & $x$ & & & \\
\hline GS & $x$ & & $x$ & & & \\
\hline VM & & $x$ & $x$ & & & $x$ \\
\hline NG & $x$ & $x$ & $x$ & & & \\
\hline PA & $x$ & $x$ & $x$ & & $x$ & \\
\hline RA & & $x$ & $x$ & & & \\
\hline RM & & & $x$ & $x$ & $x$ & $x$ \\
\hline SA & $x$ & $x$ & $x$ & $x$ & $x$ & \\
\hline GS & & $x$ & $x$ & & $x$ & \\
\hline MV & & & $x$ & & & $x$ \\
\hline SC & & $x$ & $x$ & $x$ & $x$ & \\
\hline SM & & $x$ & $x$ & $x$ & & \\
\hline TE & & $x$ & $x$ & & & \\
\hline RE & $x$ & $x$ & $x$ & $x$ & $x$ & \\
\hline GC & & $x$ & $x$ & & & \\
\hline RN & & $x$ & $x$ & & & \\
\hline PA & $x$ & $x$ & $x$ & & & $x$ \\
\hline GA & $x$ & & $x$ & $x$ & & \\
\hline
\end{tabular}

Comparison of the reference planes which have been found to be statistically significant when comparing the $3 \mathrm{D}$ technique with $2 \mathrm{D}$ technique in 20 patients.

\section{Patient BM}

The 3D versus 2D comparison assessed by Student's $t$ test where $p<0.05$ was proved to be statistically significant for the reference planes GO-ME, SNP-A and N-SNA. The 3D versus 3D comparison assessed by Student's $t$ test where $p<0.05$ was not proved to be statistically significant. The $2 \mathrm{D}$ versus $2 \mathrm{D}$ comparison was not proved to be statistically significant.

\section{Patient CF}

The 3D versus 2D comparison assessed by Student's $t$ test where $p<0.05$ was proved to be statistically significant for the reference planes GO-ME and SNP-A.

The 3D versus 3D comparison assessed by Student's $t$ test where $p<0.05$ was not proved to be statistically significant. The $2 \mathrm{D}$ versus $2 \mathrm{D}$ comparison was not proved to be statistically significant.

\section{Patient GS}

The 3D versus 2D comparison assessed by Student's $t$ test where $p<0.05$ was proved to be statistically significant for the reference planes GO-ME and S-N.

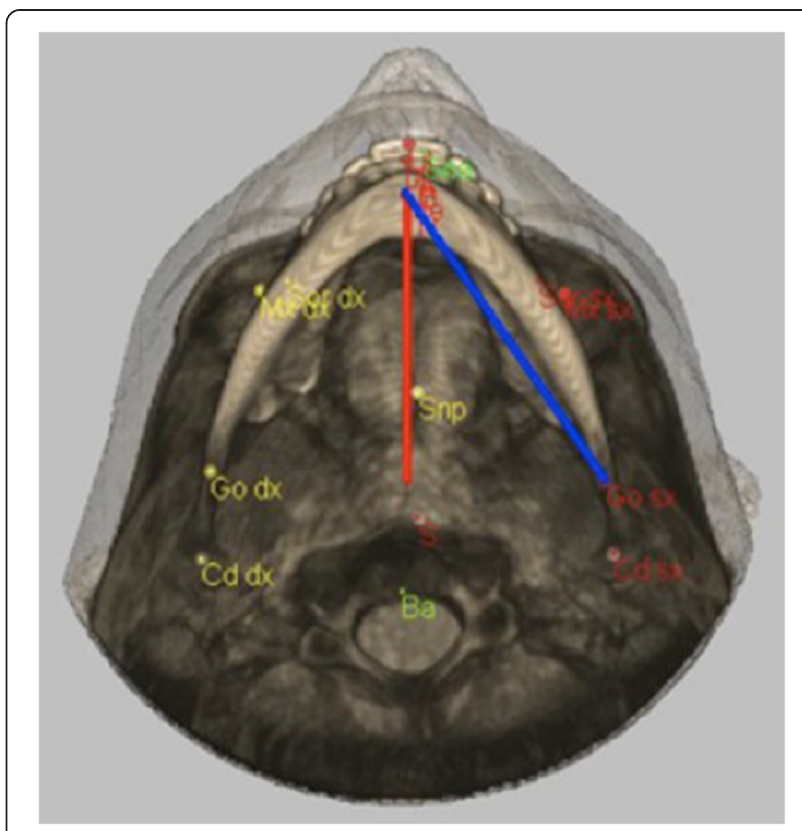

Figure 1 Reference planes: the anterior and posterior nasal spines and the GO-ME plane. The red line represents the GO-ME plane according to the $2 \mathrm{D}$ technique. The blue line represents the GO-ME plane according to the 3D technique.

The 3D versus 3D comparison assessed by Student's $t$ test where $p<0.05$ was not proved to be statistically significant. The $2 \mathrm{D}$ versus $2 \mathrm{D}$ comparison was not proved to be statistically significant.

\section{Patient MV}

The 3D versus 2D comparison assessed by Student's $t$ test where $p<0.05$ was proved to be statistically significant for the reference planes GO-ME, SNP-A and N-ME.

The 3D versus 3D comparison assessed by Student's $t$ test where $p<0.05$ was not proved to be statistically significant. The $2 \mathrm{D}$ versus $2 \mathrm{D}$ comparison was not proved to be statistically significant.

\section{Patient NG}

The 3D versus 2D comparison assessed by Student's $t$ test where $p<0.05$ was proved to be statistically significant for the reference planes GO-ME, S-N and SNP-A.

The 3D versus 3D comparison assessed by Student's $t$ test where $p<0.05$ was not proved to be statistically significant. The $2 \mathrm{D}$ versus $2 \mathrm{D}$ comparison was not proved to be statistically significant.

\section{Patient PA}

The 3D versus 2D comparison assessed by Student's $t$ test where $p<0.05$ was proved to be statistically significant for the reference planes GO-ME, S-N, SNA-ME and SNP-A. 
The $3 \mathrm{D}$ versus $3 \mathrm{D}$ comparison assessed by Student's $t$ test where $p<0.05$ was not proved to be statistically significant. The $2 \mathrm{D}$ versus $2 \mathrm{D}$ comparison was not proved to be statistically significant.

\section{Patient RA}

The 3D versus 2D comparison assessed by Student's $t$ test where $p<0.05$ was proved to be statistically significant for the reference planes GO-ME and SNP-A.

The $3 \mathrm{D}$ versus $3 \mathrm{D}$ comparison assessed by Student's $t$ test where $p<0.05$ was not proved to be statistically significant. The $2 \mathrm{D}$ versus $2 \mathrm{D}$ comparison was not proved to be statistically significant.

\section{Patient RM}

The 3D versus 2D comparison assessed by Student's $t$ test where $p<0.05$ was proved to be statistically significant for the reference planes GO-ME, SNA-ME, N-ME and N-SNA.

The $3 \mathrm{D}$ versus $3 \mathrm{D}$ comparison assessed by Student's $t$ test where $p<0.05$ was not proved to be statistically significant. The $2 \mathrm{D}$ versus $2 \mathrm{D}$ comparison was not proved to be statistically significant.

\section{Patient SA}

The 3D versus 2D comparison assessed by Student's $t$ test where $p<0.05$ was proved to be statistically significant for the reference planes GO-ME, S-N, SNA-ME, SNP-A and N-SNA.

The $3 \mathrm{D}$ versus $3 \mathrm{D}$ comparison assessed by Student's $t$ test where $p<0.05$ was not proved to be statistically significant. The $2 \mathrm{D}$ versus $2 \mathrm{D}$ comparison was not proved to be statistically significant.

\section{Patient GS}

The $3 \mathrm{D}$ versus $2 \mathrm{D}$ comparison assessed by Student's $t$ test where $p<0.05$ was proved to be statistically significant for the reference planes GO-ME, SNA-ME and SNP-A.

The $3 \mathrm{D}$ versus $3 \mathrm{D}$ comparison assessed by Student's $t$ test where $p<0.05$ was not proved to be statistically significant. The $2 \mathrm{D}$ versus $2 \mathrm{D}$ comparison was proved to be statistically significant for the reference values $\mathrm{S}-\mathrm{N}$ and N-ME.

\section{Patient MV}

The 3D versus 2D comparison assessed by Student's $t$ test where $p<0.05$ was proved to be statistically significant for the reference planes GO-ME and N-ME.

The $3 \mathrm{D}$ versus $3 \mathrm{D}$ comparison assessed by Student's $t$ test where $p<0.05$ was not proved to be statistically significant. The $2 \mathrm{D}$ versus $2 \mathrm{D}$ comparison was not proved to be statistically significant.

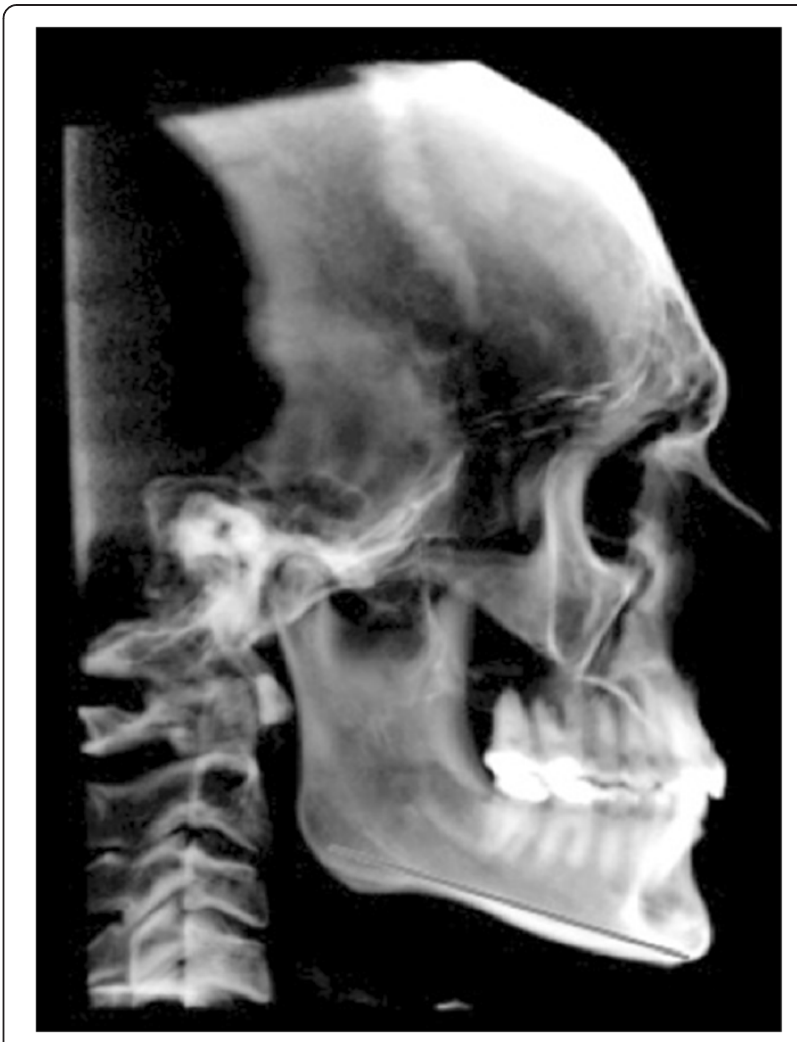

Figure 2 Latero-lateral view of the GO-ME measurement.

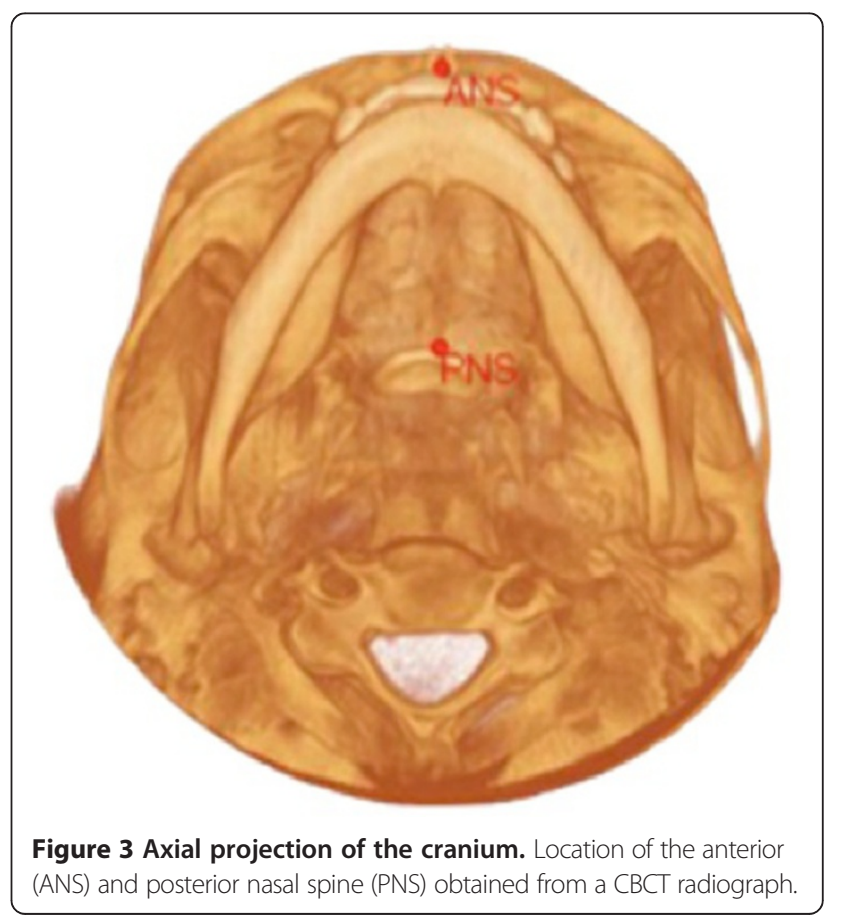




\section{Patient SC}

The 3D versus 2D comparison assessed by Student's $t$ test where $p<0.05$ was proved to be statistically significant for the reference planes GO-ME, SNA-ME, SNP-A and N-SNA.

The 3D versus 3D comparison assessed by Student's $t$ test where $p<0.05$ was not proved to be statistically significant. The $2 \mathrm{D}$ versus $2 \mathrm{D}$ comparison was proved to be statistically significant for the reference value N-ME.

\section{Patient SM}

The 3D versus 2D comparison assessed by Student's $t$ test where $p<0.05$ was proved to be statistically significant for the reference planes GO-ME, SNP-A and N-SNA.

The 3D versus 3D comparison assessed by Student's $t$ test where $p<0.05$ was not proved to be statistically significant. The $2 \mathrm{D}$ versus $2 \mathrm{D}$ comparison was proved to be statistically significant for the reference values N-ME.

\section{Patient TE}

The 3D versus 2D comparison assessed by Student's $t$ test where $p<0.05$ was proved to be statistically significant for the reference planes GO-ME and SNP-A.

Table 2 Summary of the intra-operator results for the 3D technique (3D versus 3D)

\begin{tabular}{|c|c|c|c|c|c|c|}
\hline \multirow[t]{2}{*}{ Patient } & \multicolumn{6}{|c|}{ Reference planes } \\
\hline & $\mathrm{S}-\mathrm{N}$ & SNP-A & GO-ME & N-SNA & SNA-ME & $\overline{\mathrm{N}-\mathrm{ME}}$ \\
\hline \multicolumn{7}{|l|}{$\overline{\mathrm{GA}}$} \\
\hline \multicolumn{7}{|l|}{ BM } \\
\hline \multicolumn{7}{|l|}{ CF } \\
\hline \multicolumn{7}{|l|}{ GS } \\
\hline \multicolumn{7}{|l|}{ VM } \\
\hline \multicolumn{7}{|l|}{ NG } \\
\hline \multicolumn{7}{|l|}{ PA } \\
\hline \multicolumn{7}{|l|}{ RA } \\
\hline \multicolumn{7}{|l|}{ RM } \\
\hline \multicolumn{7}{|l|}{ SA } \\
\hline \multicolumn{7}{|l|}{ GS } \\
\hline \multicolumn{7}{|l|}{ MV } \\
\hline \multicolumn{7}{|l|}{ SC } \\
\hline \multicolumn{7}{|l|}{ SM } \\
\hline \multicolumn{7}{|l|}{$\mathrm{TE}$} \\
\hline \multicolumn{7}{|l|}{ RE } \\
\hline GC & $x$ & & & & & \\
\hline \multicolumn{7}{|l|}{$\mathrm{RN}$} \\
\hline PA & & & & $x$ & & \\
\hline GA & & & & & & \\
\hline
\end{tabular}

Comparison of the reference planes, which have been found to be statistically significant when comparing the precision of the tracings in the 3D technique by the same clinicians at $\mathrm{T} 1$ and $\mathrm{T} 2$.
The 3D versus 3D comparison assessed by Student's $t$ test where $p<0.05$ was not proved to be statistically significant. The $2 \mathrm{D}$ versus $2 \mathrm{D}$ comparison was proved to be statistically significant for the reference values SNP-A and N-SNA.

\section{Patient RE}

The 3D versus 2D comparison assessed by Student's $t$ test where $p<0.05$ was proved to be statistically significant for the reference planes GO-ME, SNA-ME, SNP-A and N-SNA.

The 3D versus 3D comparison assessed by Student's $t$ test where $p<0.05$ was not proved to be statistically significant. The $2 \mathrm{D}$ versus $2 \mathrm{D}$ comparison was proved to be statistically significant for the reference values $\mathrm{S}-\mathrm{N}$ and SNP-A.

\section{Patient GC}

The 3D versus 2D comparison assessed by Student's $t$ test where $p<0.05$ was proved to be statistically significant for the reference planes GO-ME and SNP-A.

The 3D versus 3D comparison assessed by Student's $t$ test where $p<0.05$ was proved to be statistically significant

Table 3 Summary of the intra-operator results according to the $2 D$ technique (2D versus $2 D$ )

\begin{tabular}{|c|c|c|c|c|c|c|}
\hline \multirow[t]{2}{*}{ Patient } & \multicolumn{6}{|c|}{ Reference planes } \\
\hline & S-N & SNP-A & GO-ME & N-SNA & SNA-ME & $\mathrm{N}-\mathrm{ME}$ \\
\hline \multicolumn{7}{|l|}{$\overline{\mathrm{GA}}$} \\
\hline \multicolumn{7}{|l|}{ BM } \\
\hline \multicolumn{7}{|l|}{ CF } \\
\hline \multicolumn{7}{|l|}{ GS } \\
\hline \multicolumn{7}{|l|}{ VM } \\
\hline \multicolumn{7}{|l|}{ NG } \\
\hline \multicolumn{7}{|l|}{ PA } \\
\hline \multicolumn{7}{|l|}{ RA } \\
\hline \multicolumn{7}{|l|}{ RM } \\
\hline \multicolumn{7}{|l|}{ SA } \\
\hline GS & $x$ & & & & & $x$ \\
\hline \multicolumn{7}{|l|}{ MV } \\
\hline SC & & & & & & $x$ \\
\hline SM & & & & & & $x$ \\
\hline TE & & $x$ & & $x$ & & \\
\hline RE & $x$ & $x$ & & & & \\
\hline GC & & & & $x$ & & \\
\hline $\mathrm{RN}$ & & & $x$ & $x$ & & $x$ \\
\hline PA & & & & $x$ & & \\
\hline GA & & $x$ & & $x$ & & \\
\hline
\end{tabular}

Comparison of the reference planes which have been found to be statistically significant when comparing the precision of the tracings in the 2D technique by the same clinicians at $\mathrm{T} 1$ and $\mathrm{T} 2$. 
for the value S-N. The $2 \mathrm{D}$ versus $2 \mathrm{D}$ comparison was proved to be statistically significant for the values N-SNA.

\section{Patient RN}

The 3D versus 2D comparison assessed by Student's $t$ test where $p<0.05$ was proved to be statistically significant for the reference planes GO-ME and SNP-A.

The 3D versus 3D comparison assessed by Student's $t$ test where $p<0.05$ was not proved to be statistically significant. The $2 \mathrm{D}$ versus $2 \mathrm{D}$ comparison was proved to be statistically significant for the reference values GO-ME, N-ME and N-SNA.

\section{Patient PA}

The 3D versus 2D comparison assessed by Student's $t$ test where $p<0.05$ was proved to be statistically significant for the reference planes GO-ME, S-N, SNP-A and N-ME.

The 3D versus 3D comparison assessed by Student's $t$ test where $p<0.05$ was proved to be statistically significant for the value N-SNA. The $2 \mathrm{D}$ versus $2 \mathrm{D}$ comparison was proved to be statistically significant for the reference values N-SNA.

\section{Patient GA}

The 3D versus 2D comparison assessed by Student's $t$ test where $p<0.05$ was proved to be statistically significant for the reference planes GO-ME, S-N and N-SNA-ME.

The $3 \mathrm{D}$ versus $3 \mathrm{D}$ comparison assessed by Student's $t$ test where $p<0.05$ was not proved to be statistically significant. The $2 \mathrm{D}$ versus $2 \mathrm{D}$ comparison was proved to be statistically significant for the reference values SNP-A and N-SNA.

\section{Discussion}

Our findings suggest that generally the five clinicians gave a subjective interpretation of the position of the reference planes on the tracings regardless of the technique used, even though they have had the same training and had the same skills.

However, none of them obtained measurements, which were above or below the average, but they have, at times, overestimated or underestimated certain measurements when using both techniques. The comparison of the data gathered from the tracings of the $2 \mathrm{D}$ and $3 \mathrm{D}$ radiographs shows that several measurements have statistically significant differences.

Particularly, the measurement of the reference plane GOME results to be always statistically significant (Figure 2). This difference can be justified by the fact that the measurements obtained from the $2 \mathrm{D}$ technique determine a projection of the points, which in reality are placed on different planes. Differently, the planes measured using the 3D technique are based on a real measurement (an oblique plane on the sagittal axis) rather than prospective ones [6-8]. Hence, the real measurement for the two-dimensional technique is represented by its projection with the consequent result that measurements obtained from the $2 \mathrm{D}$ radiographs are less accurate than the ones obtained from the $3 \mathrm{D}$ radiographs $[19,20]$.

The other values in which we noticed a variation, which was statistically significant between the two techniques, were all values in which it is necessary to identify the anterior nasal spine (ANS) or the posterior nasal spine (PNS) (Figure 3). These points are, in fact, particularly difficult to identify with the $2 \mathrm{D}$ technique due to the numerous overlapping anatomical structures and complication, which do not occur when using the 3D technique [20].

Regarding the comparison of the linear measurements obtained by the same clinician on the tracings after 2 months, we have noticed that each clinician has traced the 2D cephalometric radiographs obtaining important variations and differences between the first time (T1) and after 2 months (T2). Each clinician has obtained, for the majority of the radiographs, the precise position of the linear measurements both times (initially and after 2 months) when tracing the 3D radiographs (Tables 2 and 3).

In conclusion, we have determined that the use of the computer for the tracings of the $3 \mathrm{D}$ radiographs obtains less measurement errors and overall much more precise cephalometric analyses. The 3D technique has thus several advantages when compared to the conventional technique such as a true representation of reality, a lesser risk of operator-dependent errors from occurring and absence of overlapping anatomical structures [21]. However, to be able to confirm such results, further research is needed on a bigger sample.

\section{Competing interests}

The authors declare that they have no competing interests.

\section{Authors' contributions}

GF supervised and coordinated the research and has given final approval of the version to be published. SS analysed the data. FN was involved in the acquisition of the data and drafting of the manuscript. AZ was involved in drafting the manuscript. DF was responsible for the statistical elaboration. All authors read and approved the final manuscript.

Received: 27 October 2013 Accepted: 12 November 2013 Published: 6 January 2014

\section{References}

1. Broadbent $\mathrm{BH}$. A new X-ray technique and its application to orthodontia. Angle Orthod. 1931; 1:45-66.

2. Downs WB. Analysis of the dentofacial profile. Angle Orthod. 1956; 26:191-212.

3. Ricketts RM. Cephalometric analysis and synthesis. Angle Orthod. 1961; 31:141-56.

4. Burstone CJ, James RB, Legan H, Murphy GA, Norton LA. Cephalometrics for orthognatic surgery. J Oral Surg. 1978; 36:269-77. 
5. McNamara JA Jr. A method of cephalometric evaluation. Am J Orthod. 1984; 86:449-69.

6. Baumrind S, Frantz RC. The reliability of head film measurements. Landmarks identification. Am J Orthod. 1971; 60:111-27.

7. Ahlqvist J, Eliasson S, Welander U. The cephalometric projection. Principles of image distortion in cephalography. Dentomaxillofac Radiol. 1983; 12:101-8.

8. Shaw K, McIntyre G, Mossey P, Menhinick A, Thomson D. Validation of conventional $2 \mathrm{D}$ lateral cephalometry using $3 \mathrm{D}$ cone beam $\mathrm{CT}$. J Orthod. 2013; 40:22-8.

9. Lou L, Lagravere MO, Compton S, Major PW, Flores-Mir C. Accuracy of measurements and reliability of landmarks identification with computed tomography (CT) techniques in the maxillofacial area: a systematic review. Oral Surg Oral Med Oral Phatol Oral Radiol Endod. 2007; 104:402-11.

10. Scarfe WC, Farman AG. What is cone-beam CT and how does it work? Dent Clin N Am. 2008; 52:707-30.

11. Ludlow JB, Davies-Ludlow LE, Brooks SL, Howerton WB. Dosimetry of 3 CBCT devices for oral and maxillofacial radiology: CB Mercuray, NewTom 3G and i-CAT. Dentomaxillofac Radiol. 2006; 35:219-26.

12. Kochel J, Meyer-Marcotty P, Strnad F, Kochel M, Stellzig-Eisenhauer A. 3D soft tissue analysis-part 1: sagittal parameters. J Orofac Orthop. 2010; 71:40-52.

13. Gribel BF, Gribel MN, Frazao DC, McNamara JA Jr, Manzi FR. Accuracy and reliability of craniometric measurements on lateral cephalometry and 3D measurements on CBCT scans. Angle Orthodontist. 2011; 81:26-35.

14. Faure J, Oueiss A, Marchal-Sixou C, Braga J, Treil J. Three-dimensional cephalometry: applications in clinical practice and research. Orthod Fr. 2008; 79(1):13-30.

15. Farronato G, Maspero $C$, Farronato D. Orthodontic treatment in a patient with cleidocranial dysostosis. Angle Orthodontist. 2009; 79:178-85.

16. Farronato G, Carletti V, Maspero C, Farronato D. Craniofacial growth in children affected by juvenile idiopathic arthritis involving the temporomandibular joint: functional therapy management. I Clin Pediatr Dentistry. 2009; 33:351-7.

17. Kochel J, Meyer-Marcotty P, Kochel M, Schneck S, Stellzig-Eisenhauer A 3D soft tissue analysis-part 2: vertical parameters. J Orofac Orthop. 2010; 71:207-20.

18. Silva MA, Wolf U, Heinicke F, Bumann A, Visser H, Hirsch E. Cone-beam computed tomography for routine orthodontic treatment planning: a radiation dose evaluation. Am J Orthod Dentofacial Orthop. 2008; 133:640-5.

19. Farronato $G$, Farronato $D$, Toma $L$, Bellincioni F. A synthetic three-dimensional craniofacial analysis. J Clin Orthod. 2010; 44:673-8.

20. Farronato G, Garagiola U, Dominici A, Periti G, de Nardi S, Carletti V, Farronato D. "Ten-point" 3D cephalometric analysis using low-dosage cone beam computed tomography. Prog Orthod. 2010; 11:2-12.

21. Agrawal JM, Agrawal MS, Nanjannawar LG, Parushetti AD. CBCT in orthodontics: the wave of future. J Contemp Dent Pract. 2013; 14:153-7.

doi:10.1186/2196-1042-15-1

Cite this article as: Farronato et al:: Assessment of inter- and intraoperator cephalometric tracings on cone beam $\mathrm{CT}$ radiographs: comparison of the precision of the cone beam CT versus the laterolateral radiograph tracing. Progress in Orthodontics 2014 15:1.

\section{Submit your manuscript to a SpringerOpen ${ }^{\circ}$ journal and benefit from:}

- Convenient online submission

- Rigorous peer review

- Immediate publication on acceptance

- Open access: articles freely available online

- High visibility within the field

- Retaining the copyright to your article

Submit your next manuscript at $\gg$ springeropen.com 